\title{
Integrated circuit system design based on "PLC" programming technology and the development of the device which can monitor the concentration of pollutants continuously on-line
}

\author{
Yuchuan Huang \\ School of North China Electric Power University, Baoding 071000, China
}

Keywords: Online data acquisition system, power, serial port communication, algorithm design, integrated circuit system, PLC programming

\begin{abstract}
In China, the number of the power station increases quickly. According to the information from the Internet, From 2001 to August 2005, State Environmental Protection Administration has approved 472 thermal power projects, and the installed capacity is as much as 344382MW.In which , 135 thermal power projects are approved in 2001, the installed capacity is as much as $107590 \mathrm{MW}, 207 \%$ more than last year; 213 thermal power projects are approved From January to August in 2005, the installed capacity is as much as $168546 \mathrm{MW}, 420 \%$ more than last year.If these power stations all come online, Chinese installed capacity will be as much as 5.82 billion KW, $145 \%$ more than 2000. In December 2006 , the growth of the power stations keeps high levels. The same month, the whole country's generating capacity was 2266 billion kilowatt-hour, $15.5 \%$ more than last year.As the rise of the winter electricity consumption, the generating capacity of power projects increased quickly.The genearating capacity of power projects in December was $10.9 \%$ more than last month.From January to December in 2006 the whole country's generating capacity was 23009 billion kilowatt-hour, 15.08\% more than last year.From January to October in 2007 the whole country's generating capacity was 21756 billion kilowatt-hour, $16.04 \%$ more than last year.The generating capacity in August had the largest number, which was 2390 billion kilowatt-hour, 10.19\% more than last year.
\end{abstract}

\section{Introduction}

The growth of the power station gives people rich electric power, but at the same time it also has big bad effect on the environment.Chinese air pollution status is un-unfavorable, which marked by coal smoke pollution. The increase of the number of power stations leads to the increase of the consumption of the coal, which causes emissions of sulfur dioxide sharply increase.And the emissions from power station and the boiler is equal to about $70 \%$ of all kinds of emissions. The emissions of sulfur dioxide caused acid rain.And the acid rain's sphere of influence has been more and more large.Now the pollution of the acid rain has extended to the most areas in the south of Changjiang River and the east of Qinghai-Tibetan Plateau.The areas whose PH value of the rainfall is below the critical value of the acid rain's has a very large acreage, which has accounted for $30 \%$ of the whole country.According to the budget, there will be a large increase in the development, utilization and the dosage of the energy.And Chinese economic development level and its energy characteristic determined that the coal will be the main energy source to China for a long time.So controlling the air pollution due to coal combustion has great economic benefits and social effectresults benefits.In order to control the emissions of the pollutants from the power station, knowing the accurate information of the power station is very important.According to the information we get, we can control the power station better, and then we can advance the efficiency of the power station and reduce the pollution of the power station.

In order to get the information of the power station, we need build a online data acquisition system, then I will introduce one kind of the online data acquisition system of the power station network information management system that under the client / server mode.In this system the data acquisition computer should write all the data into the serves every 5seconds, the severs keep the data for 6 days and the serves delete the 6-day-ago data every once in a while. This system's client computer development platform is Windows 98, And the development tool is Delphi 4.0.The system 
of the servers is Windows NT and the databases is Oracle 7.3.This system's data acquisition and communication program uses the Communication Control --MS COMM.Using the MS COMM to accomplish the serial communication has the advances as bellow:Firstly, the program can nearly realize all the functions of the Windows API.Secondly, the efficiency of writing program is high.the MS COMM has two kinds of communication methods.One is the query mode, this kind of mode through detecting the attribute value of the CommEvent to inquire the generation of the communication event and exception. This kind of mode is usually used in the simple or independent programming design.And the other is the event-driven mode.The trigger of the Oncomm event can deal with the generation of the communication event and exception, which can be achieved by programming every attribute of the CommEvent.This kind of mode has powerful function. The system here chooses the second mode.

The main control computer is the computer that monitor the running conditions of all the set. When the system running, the set's running parameters and indicator data will be sent to the data acquisition computer.And the data acquisition computer should sort and write the data into the homologous buffer.Then the data acquisition computer should write the data to the database in the severs every 5 seconds.At the same time the computer should inspect the important indicators of the generator set.If the indicators are beyond the limit, the computer should send the information of alarm.

The communication part of the data acquisition system should be written in three parts:

\section{The initialization of the MSCOMM control}

The list of the program and the annotations is as below:

//Initialize the MS Comm 1

with MS Comm 1 do //the MS Comm is the name of the control

begin

Comm Port : $=1 ; \quad / /$ the serial port COM1

InBufferSize : = 1024; //set the magnitude of the receive buffer is 1024 bytes

InputLen : $=0 ; \quad / /$ read all the characters of the receive buffer

InputMode : $=1 ; \quad / /$ receive in binary

RThreshold : $=128 \quad / /$ set the data acquisition

triggering the OnComm event when the computer receives every 128 Bytes

Settings : ='9600, n, 8, 1'; //set the baud rate is 9600 , no check bit, the date bits is 8 , the stop bits is 1

end;

StopCJ.Enabled : = FLASE; //do not allow stop acquiring

StartFlag: =FLASE; //the data acquisition is no begin

The MS Comm 1 control can work according to the set after initializing.The control can receive the operating parameters and the performance indexes of the set, and trigger the OnComm event.

\section{OnComm event}

When the number of characters of the serial port receiving buffer reach the attribute that have been set of the RThreshold will trigger the OnComm event.Receiving and processing the data in the interrupt routine. The list of the program and the annotations is as below:

procedure TMainForm.MS Comm 1 Comm(Sender:TObject); Var

IArray :Variant; //read the array of values

RecCount, I:Integer; //input the number of the buffer characters

Frame Array:Array 0..1023 O F Byte; //displays the string 
begin

MsgEvent: string; //the information of the event

MsgError:='Acquiring';

Case MS Comm 1.Comm Event of com EvReceive: //receive data

begin

MsgEvent:='Receiving Data';

IArray:=VarArrayCreate(0, 1023, var 2 Byte); //binding array

RecCount :=MS Comm 1.InBufferCount; //read the number of the input buffer characters

MS Comm 1.InputI.en:=RecCount;

IArray:=MS Comm 1.Input; //read the input frames

for $1:=0$ to RecCount -1 do

begin

end;

FrameArrayI:=IArrayI;

Write To Data Buf(FrameArray, RecCount); //write the receiving data into the creating buffer

end;

comEvSend:

MsgEvent :='There are characters in send buffer';

comTxFull:

MsgEvent:='The send buffer is full';

Else

MsgEvent:='Unkow error or event';

end;

StatusBarl.Panels I.text='The current state:'+ MsgEvent;

end;

\section{Timer program}

The interrupt program of the timer deals with the data in the creating buffer and writes the data into the severs every 5 seconds.the flow chart of the program is as bellow: 


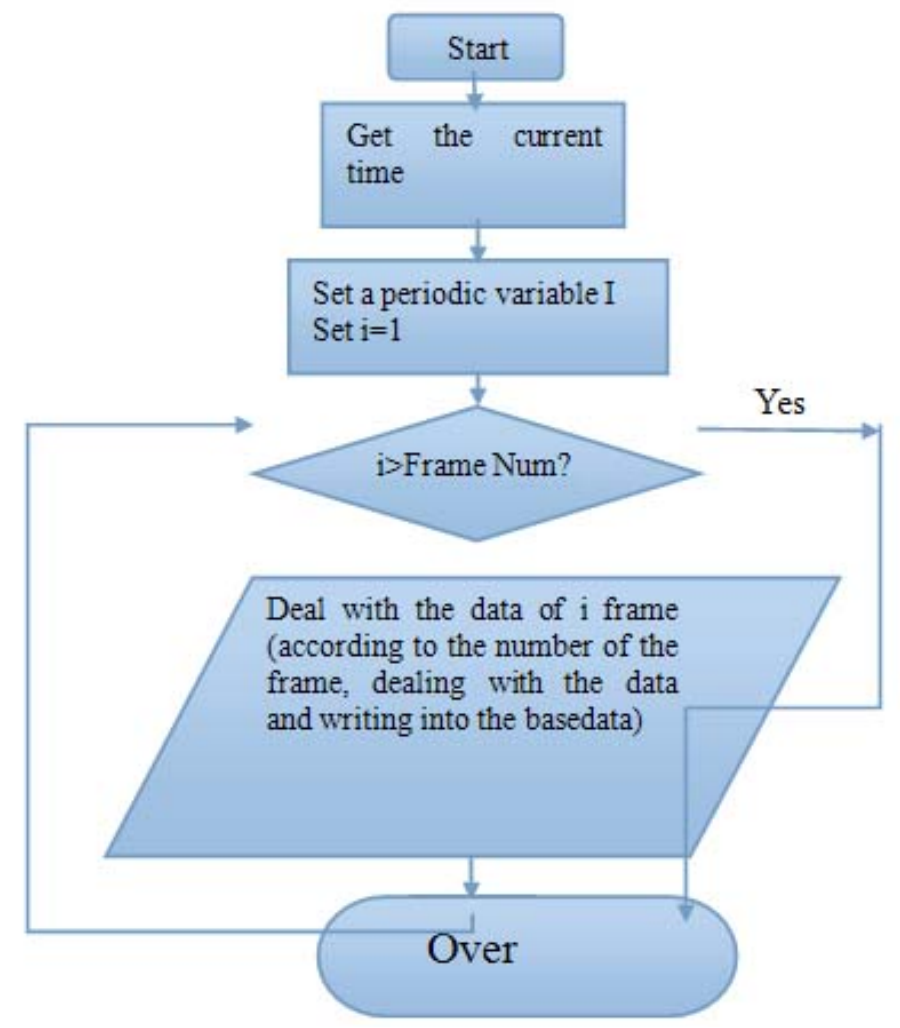

In order to make the serves keep the data of the set for 6 days, I define a job in the Oracle database.The definition of the job is as below:

VARIABLE V-JobNum NUMBER ;

BEGIN

DBMS-JOB 1 SUBMIT（: V-JobNum, 'AIDellAll;'SYSDATE, ”Sysdate + 1/24' ）;

END;

BEGIN

DBMS - JOB 1 CHANGE(101, ’AIDelall;', SYSDATE, ”Sysdate + 1/24');

END

CREATE OR REPLACE PROCEDURE AIDelAll AS V CurTime DATE;

BEGAIN

V-CurTime :=sysdate;

COMMIT;

END AIDelAll;

Nowadays, with the development of the power station, an accurate and stable online data acquisition system is being more and more necessary.An good stable online data acquisition system can help people control the power station better, and then people can not only get benefit but also can protect the environment.So the system put forward in this article is feasible and promising.

\section{References}

[1] N. Koutsouris, K. Tsagkaris, P. Demestichas, L. Mamatas, S. Clayman, and A. Galis, Managing Software-Driven Networks with a unified management framework, in Integrated Network Management (IM 2013), 2013 IFIP/IEEE International Symposium on, 2013, pp. 1084-1085.

[2] A. R. Sharafat, S. Das, G. Parulkar, and N. McKeown, MPLS-TE and MPLS VPNS with openflow, SIGCOMM Comput. Commun. Rev., vol. 41, pp. 452-453, 2011. 
[3] A. Lara, A. Kolasani, and B. Ramamurthy, Simplifying network management using Software Defined Networking and OpenFlow, in Advanced Networks and Telecommuncations Systems (ANTS), 2012 IEEE International Conference on, 2012, pp. 24-29.

[4] D. M. F. Mattos, N. C. Fernandes, V. T. da Costa, L. P. Cardoso, M. E. M. Campista, L. H. M. K. Costa, et al., "OMNI: OpenFlow MaNagement Infrastructure," in Network of the Future (NOF), 2011 International Conference on the, 2011, pp. 52-56.

[5] Z. A. Qazi, C.-C. Tu, L. Chiang, R. Miao, V. Sekar, and M. Yu, "SIMPLE-fying middlebox policy enforcement using SDN," presented at the Proceedings of the ACM SIGCOMM 2013 conference on SIGCOMM, Hong Kong, China, 2013. 